\title{
Lung Metastases Versus Second Primary Lung Cancers in Patients with Primary Urothelial Carcinoma of the Bladder: A National Population-Based Assessment
}

Jacob Taylor ${ }^{\mathrm{a}, *}$, Adam B. Weiner ${ }^{\mathrm{b}}$, Binhuan Wang ${ }^{\mathrm{c}}$, Arjun V. Balar ${ }^{\mathrm{d}}$, Gary D. Steinberg ${ }^{\mathrm{a}}$ and Richard S. Matulewicz ${ }^{\mathrm{a}, \mathrm{c}}$

${ }^{a}$ Department of Urology, NYU Grossman School of Medicine, New York, NY, USA

${ }^{\mathrm{b}}$ Department of Urology, Northwestern University, Feinberg School of Medicine, Chicago, IL, USA

${ }^{\mathrm{c}}$ Department of Population Health, NYU Grossman School of Medicine, New York, NY, USA

${ }^{\mathrm{d}}$ Department of Medicine, NYU Grossman School of Medicine, Perlmutter Cancer Center, New York, NY, USA

Received 18 January 2021

Accepted 5 June 2021

Pre-press 22 June 2021

Published 31 August 2021

\begin{abstract}
.
BACKGROUND: The work-up and diagnosis of indeterminate lung nodules at time of bladder cancer diagnosis may delay or change treatment.

OBJECTIVE: To quantify the incidence of synchronous and metachronous lung cancers in adults with bladder cancer and compare these rates to the incidence of bladder cancer metastases in the lung.

METHODS: We retrospectively analyzed all adults diagnosed with bladder cancer in the Surveillance, Epidemiology and End Results (SEER) registry (2010-2015) and identified second primary lung cancers defined as being either synchronous (diagnosed within 6 months of bladder cancer diagnosis) or metachronous (more than 6 months following index bladder cancer diagnosis). The risk of second primary lung cancers were reported as a standardized incidence ratio (SIR) reflecting observed and expected case ratios.

RESULTS: A total of 88,335 patients diagnosed with bladder cancer were included. Among adults with NMIBC $(n=66,071)$ and $\operatorname{MIBC}(n=18,879), 0.3 \%$ and $3.9 \%$ had bladder cancer metastatic to the lungs at diagnosis. Synchronous second primary lung cancers were diagnosed in $0.4 \%$ and $0.7 \%$ of patients with NMIBC and MIBC, respectively. Compared to the general population, the SIR for synchronous lung cancers among adults with NMIBC was 2.5 (95\% CI 2.3-2.9) and was 4.7 (95\% CI 4.0-5.6) for adults with MIBC.

CONCLUSIONS: Bladder cancer metastatic to the lung is more common in adults with MIBC compared to NMIBC. There are similar frequencies of synchronous second primary lung cancers regardless of initial bladder cancer stage.
\end{abstract}

Keywords: Urinary bladder neoplasms, neoplasms, multiple primary, neoplasms, second primary, lung neoplasms, early detection of cancer

\footnotetext{
*Correspondence to: Jacob Taylor, MD, MPH, Department of Urology, 222 E 41st, New York, NY 10017, USA. Tel.: +001 781

789 7047; E-mail: Jacob.taylor@nyulangone.org.
} 


\section{INTRODUCTION}

At the time of a new bladder cancer diagnosis, it is common to discover indeterminate lung lesions on staging imaging. These findings may represent the presence of metastatic bladder cancer or a second primary lung cancer. These difficult clinical conundrums usually require further diagnostic pursuit, potentially including a biopsy or interval imaging. This subsequent evaluation may delay definitive treatment which is critical to good outcomes in aggressive bladder cancer [1,2]. An additional consideration is how diagnosing a second primary cancer may also lead to considerable changes in the original bladder cancer treatment plan.

In general, cancer survivors are at an increased risk of developing second primary malignancies, particularly when cancers share common risk-factors, such as tobacco use [3]. An estimated 712,000 bladder cancer patients in the US will be at risk for second cancers as survival increases with improved bladder cancer treatments [4]. Timely diagnosis of second malignancies is critical for improving survivorship and decreasing morbidity and mortality from secondary disease [5].

Unfortunately, despite the causal link between smoking and both lung and bladder cancer [6-8], the comparative incidence of metastatic bladder and synchronous second primary cancers is poorly quantified as is the timing and incidence of metachronous lung cancers after a diagnosis of bladder cancer. We hypothesize that there is an underappreciated risk of second primary lung cancers at both the time of diagnosis and long term for adults with bladder cancer. Therefore, the aim of this study is to use a national, population-level dataset to evaluate and compare the rates of metastatic bladder cancer with synchronous and metachronous lung cancers to help inform patient counseling and guide clinical decision making.

\section{METHODS}

\section{Data source}

This is a retrospective cohort study using the population-level cancer registry data included in the National Cancer Institute's Surveillance, Epidemiology, and End Results (SEER) 18 registries [4]. This resource collects data on approximately $34.6 \%$ of the United States population and is considered to be broadly generalizable to the national population [9]. Using SEER*Stat version 8.3.6 [10], we searched for all adults (age 18 and older) diagnosed with urinary bladder cancer as their first malignant primary tumors in the SEER database between 2010 and 2015. We excluded adults diagnosed with bladder cancer from autopsy or death certificate alone. Follow-up for this cohort could extend as late as December 31, 2016.

\section{Variable definitions}

Age was categorized as $18-59,60-69,70-79$, and 80 years and older. Gender (male or female), marital status (married, not married, or unknown), and race (Non-Hispanic White, Non-Hispanic Black, NonHispanic Asian Pacific Islander, and Hispanic, or other/unknown) were also included as demographic variables. Cancer staging characteristics were determined according to the American Joint Committee on Cancer staging criteria [11]. Primary analyses were conducted on two cohorts stratified by disease status: those with non-muscle invasive bladder cancer (NMIBC, $<$ pT2) and those with muscle invasive bladder cancer (MIBC, $\geq \mathrm{pT} 2$ ). This stratification was performed to account for the inherent differences in the risk of metastatic disease at diagnosis as well as disparate recommendations and practices related to imaging intensity (e.g. chest $\mathrm{CT}$ vs. $\mathrm{x}$-ray). Nodal stage was categorized as either NO (no nodal involvement), $\mathrm{N}+$ (nodal involvement), or Nx (unable to determine). Metastatic disease was determined to be either M0 (no metastatic disease), M1 (metastatic disease present) or Mx (unable to determine). M1 patients were further divided by the presence of lung metastases as either present, absent, or unable to be determined. All included diagnoses were histologically confirmed.

Synchronous and metachronous second malignant primary cancers were defined as being diagnosed within 6 months of diagnosis (synchronous) and 6 months or more (metachronous) following the diagnosis of the index bladder cancer according to accepted SEER definitions [12]. Our outcomes of interest were incidence of metastatic bladder cancer to the lung at the time of diagnosis, and incidences and timing (either synchronous or metachronous) of second primary lung cancer among patients with a primary diagnosis of bladder cancer.

\section{Statistical analysis}

Covariates within each cohort were reported as percentages of total patients. The risk of second primary lung cancers was calculated using SEER*Stat 
Table 1

Sociodemographic characteristics, tumor staging, and synchronous lung cancer incidence among adults diagnosed with bladder cancer 2010-2015 $(N=84,950)$ stratified by muscle-invasion status

\begin{tabular}{llcc}
\hline & & NMIBC $(n=66,071)$ & MIBC $(n=18,879)$ \\
\hline Age (years) & $18-59$ & $\mathrm{~N}(\%)$ & $\mathrm{N}(\%)$ \\
& $60-69$ & $11,860(18.0)$ & $3,101(16.4)$ \\
& $70-79$ & $18,026(27.3)$ & $4,810(25.5)$ \\
Gender & $80+$ & $19,433(29.4)$ & $5,292(28.0)$ \\
& Male & $16,752(25.4)$ & $5,676(30.1)$ \\
Marital Status & Female & $50,550(76.5)$ & $13,497(71.5)$ \\
& Married & $15,521(23.5)$ & $5,382(28.5)$ \\
Race/Ethnicity & Not married & $38,678(58.5)$ & $9,983(52.9)$ \\
& Unknown & $20,974(31.7)$ & $8,013(42.4)$ \\
& Non-Hispanic White & $6,419(9.7)$ & $883(4.7)$ \\
& Non-Hispanic Black & $54,586(82.6)$ & $15,114(80.1)$ \\
& Asian/Pacific Islander & $3,252(4.9)$ & $1,489(7.9)$ \\
N-Stage & Hispanic & $2,790(4.2)$ & $833(4.4)$ \\
& Other/unknown race & $4,132(6.3)$ & $1,329(7.0)$ \\
& No & $1,311(2.0)$ & $114(0.6)$ \\
M-stage & N+ & $65,248(98.8)$ & $14,850(78.7)$ \\
& Nx & $293(0.4)$ & $3,380(17.9)$ \\
Lung metastasis & M0 & $530(0.8)$ & $649(3.4)$ \\
& M1 & $65,294(98.8)$ & $16,416(87.0)$ \\
Synchronous Lung cancer & Mx & $519(0.8)$ & $2,199(11.6)$ \\
& None & $258(0.4)$ & $264(1.4)$ \\
& Present & $65,627(99.3)$ & $17,876(94.7)$ \\
& Unknown & $196(0.3)$ & $733(3.9)$ \\
None & $248(0.4)$ & $270(1.4)$ \\
& Present & $65,793(99.6)$ & $18,747(99.3)$ \\
& & $278(0.4)$ & $132(0.7)$ \\
\hline
\end{tabular}

NMIBC: non-muscle invasive bladder cancer; MIBC: muscle-invasive bladder cancer.

and reported as a standardized incidence ratio (SIR) reflecting observed and expected case ratios $(\mathrm{O} / \mathrm{E}$ ratio). The number of observed cases was the actual incidence among the at-risk group of patients. The number of expected cases was based on a representative sample of the general population with analogous demographic information (race and age) over a similar follow-up period. Patients were removed from the at-risk group when they were diagnosed with a second primary lung malignancy. SIR $95 \%$ confidence intervals (CIs) were calculated using exact methods. Statistical significance for SIRs were based on $p$ values generated from 2 -sided tests. SIRs and $p$-value calculations were suppressed for groups of $<10$ men in the cohort. Excess incidence risk was calculated within SEER* ${ }^{*}$ stat as the difference between observed and expected incidences per 10,000 person years. No adjustments for multiple hypothesis testing were performed due to the exploratory nature of this study. Sensitivity analyses were performed with varying designations for synchronous and metachronous time cut points to assess for robustness of our analysis. Data were acquired and approved by NCI SEER Program ID: 20565-Nov2018. Our study was determined by the New York University Institutional Review
Board to be exempt since the work did not involve human subjects.

\section{RESULTS}

\section{Cohort characteristics}

A total of 88,335 patients diagnosed with bladder cancer as their first primary malignancy between 2010 and 2015 were identified and met inclusion criteria. 84,950 patients remained after excluding those with missing tumor staging of the index bladder cancer. The majority $(77.8 \%, n=66,071)$ of included patients had NMIBC. There was a male predominance in both the NMIBC and MIBC cohorts. Patients with MIBC were generally older (Table 1).

\section{Incidence of metastatic bladder cancer vs. synchronous second primary lung cancer}

Among adults with NMIBC, $0.8 \%(n=519)$ had metastatic bladder cancer and $0.3 \%(n=196)$ had bladder cancer metastatic to the lungs at diagnosis. Accordingly, $0.4 \%(n=278)$ had a synchronous lung cancer diagnosis. For those with MIBC, $11.6 \%$ 
$(n=2,199)$ had metastatic bladder cancer at diagnosis and $3.9 \%(n=733)$ had bladder cancer metastases in the lungs. Among adults with MIBC, $0.7 \%(n=132)$ were diagnosed with a synchronous lung cancer (Table 1).

\section{Incidence ratios for synchronous and metachronous lung cancers}

Standardized incidence ratios for synchronous and metachronous lung cancers are found in Table 2. Compared to a general population, there was a 2.5 times increased risk of synchronous lung cancers among adults with NMIBC (SIR 2.5, 95\% CI 2.3-2.9) and an even higher risk for MIBC (SIR 4.7, 95\% CI 4.0-5.6). This amounted to an excess risk of 46 and 109 synchronous lung cancers per 10,000

Table 2

Observed to expected and standardized incidence ratios of synchronous and metachronous lung cancers diagnosed after a bladder cancer diagnosis

\begin{tabular}{cccc}
\hline & $\begin{array}{c}\text { Observed/ } \\
\text { Expected }\end{array}$ & SIR $(\mathrm{CI})$ & $\begin{array}{c}\text { Excess risk } \\
\text { per 10,000 } \\
\text { people) }\end{array}$ \\
\hline Synchronous $(<6 \mathrm{~m})$ & & & \\
NMIBC $(N=66071)$ & $278 / 109$ & $2.5(2.3-2.9)^{*}$ & 46 \\
MIBC $(N=18879)$ & $132 / 28$ & $4.7(4.0-5.6)^{*}$ & 109 \\
Metachronous $(\geq 6 \mathrm{~m})$ & & & \\
NMIBC $(N=62161)$ & $1065 / 542$ & $2.0(1.9-2.1)^{*}$ & 29 \\
MIBC $(N=14261)$ & $177 / 79$ & $2.2(1.9-2.6)^{*}$ & 36 \\
\hline
\end{tabular}

O/E: number of observed cases/number of expected cases; SIR: standardized incidence ratio; ${ }^{*} p=<0.05$. person-years in adults with NMIBC and MIBC, respectively, compared to adults with similar demographics characteristics but without bladder cancer. The risks of metachronous ( $>6$ months) second primary lung cancers were also higher than the general population for adults with both NMIBC (SIR 2.0, 95\% CI 1.9-2.1) and MIBC (SIR 2.2, 95\% CI 1.9-2.6). Supplementary Table 1 shows stratification of synchronous and metachronous lung cancers by additional time cut points and indicates the highest risk for synchronous cancers occurs during months 1-3 after bladder cancer diagnosis for MIBC patients.

Further stratifying adults according to age and gender determined that certain subgroups had a substantially higher risk for synchronous or metachronous lung cancers than others (Table 3 ). The risk of synchronous lung cancers was highest among younger adults ages $18-59$ in the case of both NMIBC (SIR 4.3, 95\% CI 2.5-6.8) and MIBC (SIR 9.6, 95\% CI 4.6-17.6). Patients with MIBC ages 18-59 also had a 5.5 times increased risk of metachronous lung cancers. Females across bladder cancer stage saw higher risks of synchronous and metachronous cancers compared to males. Patients with high grade NMIBC had a higher risk of synchronous lung cancers.

\section{DISCUSSION}

Using a large population-based cancer registry we determined that in adults with MIBC, there is a

Table 3

Observed, expected, and excess risk of synchronous and metachronous lung cancers stratified by bladder cancer T-stage and sub-stratified by age, gender, and pathologic grade

\begin{tabular}{|c|c|c|c|c|c|c|c|c|c|}
\hline & & \multicolumn{4}{|c|}{ Synchronous $(<6 \mathrm{~m})$} & \multicolumn{4}{|c|}{ Metachronous $(\geq 6 \mathrm{~m})$} \\
\hline & & Observed & Expected & $\operatorname{SIR}(95 \% \mathrm{CI})$ & $\begin{array}{c}\text { Excess } \\
\text { Risk }\end{array}$ & Observed & Expected & $\operatorname{SIR}(95 \% \mathrm{CI})$ & $\begin{array}{c}\text { Excess } \\
\text { Risk }\end{array}$ \\
\hline \multirow[t]{9}{*}{ NMIBC } & All & 278 & 109.3 & $2.5(2.3-2.9)^{*}$ & 46 & 1,065 & 541.6 & $2.0(1.9-2.1)^{*}$ & 29 \\
\hline & $18-59$ & 17 & 4 & $4.3(2.5-6.8)^{*}$ & 19 & 85 & 29.1 & $2.9(2.3-3.6)^{*}$ & 15 \\
\hline & $60-69$ & 70 & 21.6 & $3.2(2.5-4.1)^{*}$ & 47 & 294 & 135.9 & $2.2(1.9-2.4)^{*}$ & 29 \\
\hline & $70-79$ & 101 & 44.6 & $2.3(1.9-2.8)^{*}$ & 51 & 445 & 227.3 & $2.0(1.8-2.2)^{*}$ & 40 \\
\hline & $80+$ & 90 & 39.1 & $2.3(1.9-2.8)^{*}$ & 56 & 241 & 149.3 & $1.6(1.4-1.8)^{*}$ & 25 \\
\hline & Male & 217 & 89.6 & $2.4(2.1-2.8)^{*}$ & 45 & 846 & 442.5 & $1.91(1.8-2.1)^{*}$ & 29 \\
\hline & Female & 61 & 19.7 & $3.1(2.4-4.0)^{*}$ & 48 & 219 & 99.2 & $2.2(1.9-2.5)^{*}$ & 28 \\
\hline & High Grade & 119 & 41 & $2.9(2.4-3.5)^{*}$ & 59 & 367 & 180 & $2.03(1.83-2.25)^{*}$ & 32 \\
\hline & Low Grade & 93 & 38 & $2.43(1.96-2.98)^{*}$ & 39 & 390 & 193 & $2.03(1.83-2.24)^{*}$ & 28 \\
\hline \multirow[t]{7}{*}{ MIBC } & All & 132 & 28 & $4.7(4.0-5.6)^{*}$ & 109 & 177 & 79.3 & $2.23(1.9-2.6)^{*}$ & 36 \\
\hline & $18-59$ & 10 & 1.0 & $9.6(4.6-17.6)^{*}$ & 54 & 25 & 4.5 & $5.56(3.6-8.2)^{*}$ & 37 \\
\hline & $60-69$ & 40 & 5.3 & $7.5(5.4-10.2)^{*}$ & 136 & 51 & 20.7 & $2.46(1.8-3.2)^{*}$ & 36 \\
\hline & $70-79$ & 45 & 10.9 & $4.2(3.1-5.6)^{*}$ & 126 & 71 & 32.5 & $2.19(1.7-2.8)^{*}$ & 49 \\
\hline & $80+$ & 37 & 10.8 & $3.4(2.4-4.7)^{*}$ & 101 & 30 & 21.6 & $1.39(0.9-2.0)$ & 16 \\
\hline & Male & 87 & 21.9 & $4.0(3.2-4.9)^{*}$ & 94 & 133 & 63.9 & $2.08(1.7-2.5)^{*}$ & 34 \\
\hline & Female & 45 & 6.0 & $7.5(5.4-10.0)^{*}$ & 149 & 44 & 15.4 & $2.85(2.1-3.8)^{*}$ & 42 \\
\hline
\end{tabular}

${ }^{*} p=<0.05, \mathrm{O}=$ observed, $\mathrm{E}=$ expected; SIR $=$ standardized incident ratio; Excess risk is per 10,000 person-years. 
roughly five times greater incidence of lung metastases from bladder cancer (3.9\%) than synchronous second primary lung cancers $(0.7 \%)$ near the time of diagnosis. This information may help inform the decision making process when weighing the risks and benefits of further diagnostic evaluation of lung masses at the time of MIBC diagnosis. Accordingly, among adults with NMIBC, we found a similar incidence of bladder cancers metastatic to the lung $(0.3 \%)$ compared with synchronous second primary lung cancers $(0.4 \%)$.

The disparate rates of lung metastases for MIBC (3.9\%) and NMIBC $(0.3 \%)$ are likely the result of the biologic potential of more aggressive disease to spread wider in the body. However, the similar incidence of synchronous primary lung cancers regardless of bladder cancer stage $(0.7 \%$ and $0.4 \%)$ may be due to either a true, similar risk of a second primary lung cancer, or may be related to differences in the approach to staging. Adults with NMIBC likely receive less intensive chest imaging (Chest X-Ray vs. Computed Tomography scan) than those with MIBC. This calls into question how variation in the utilization of lung imaging at bladder cancer diagnosis can influences detection rates and is an important potential area for subsequent research.

Another important finding of our study was quantifying the increased risk of metachronous second lung cancers among bladder cancer patients compared to a general population, particularly among individual age and gender subgroups. The increased SIR among younger adults $(<60)$ with both NMIBC and MIBC may support efforts that selectively screen this highrisk group of bladder cancer survivors for lung cancer. Timely diagnosis of second malignancies is critical for improving survivorship and decreasing morbidity and mortality from secondary disease [5]. Alternatively, the higher risk of subsequent lung cancers in younger adults may be related to a competing risk of mortality from bladder cancer or other causes in the elderly.

Although most previous studies have been in small cohorts or have focused on non-muscle invasive bladder cancer (NMIBC) and the incidence of second cancers [13-15], our findings are similar to data from French cancer registries on 11,000 patients diagnosed with bladder cancer with SIRs for lung cancer of 3.12 and 3.27 for men and women, respectively [16]. Our data demonstrated higher SIRs among females across tumor stage and time to lung cancer development, with the highest rate 7.5 for synchronous lung cancers in females with MIBC. This is in contrast to historical data and higher rates of lung and bladder cancer in men [17]. These data are also consistent with prior SEER registry data from 1993 - 2002 that found an SIR of 1.71 for second lung cancers after NMIBC [14]. Although this study found a cumulative 15 -year incidence of $8.8 \%$ compared to $4.3 \%$ expected, the study did not include MIBC patients or designate a difference in the risk of synchronous versus metachronous disease. A separate case series from Spain suggest a 10-fold higher rate of second lung cancers at a median interval occurrence of 54.2 months in NMIBC patients [13]. This contrasts with data from Kwon et al. who did not find increases in overall second lung cancers after bladder cancer diagnosis [18].

Cancers of the bladder and lung share a common causal etiology [7] and recognition of their connection is underappreciated. In addition to these data, prior work has shown a pronounced risk of second primary cancers within the first 2-3 years after cancer diagnosis [19], suggesting that a more complete upfront staging for NMIBC may be warranted. Furthermore, one tumor registry indicates worse survival in patients diagnosed with both cancers when bladder is diagnosed before lung (17 vs. 42 months) [20]. In a separate small series, lung cancer was the cause of death in all 10 patients who developed both NMIBC and lung cancer [13], indicating lung cancer drives mortality when both cancers are diagnosed. This highlights the potential for guidelines to include staging protocols that specifically assess for lung cancer in high-risk patients. A second primary lung cancer could change the overall treatment approach for a patient with bladder cancer who may have otherwise been treated locally for NMIBC or with systemic chemotherapy for presumed metastatic muscle invasive bladder cancer. Further studies are warranted to determine if intensive lung cancer screening can improve outcomes for patients with NMIBC. Screening protocols could be tailored to higher risk groups based on age, gender, smoking risk, and interval from initial cancer diagnosis. Regular screening for lung cancer could also serve as additional opportunities for urologists to engage in targeted screening efforts and smoking cessation education for bladder cancer patients who continue to smoke.

Limitations of the data include the lack of smoking information or subsequent metastatic bladder cancer risk in our analysis, as these variables are not available in the SEER dataset. Smoking could potentially confound the development of secondary lung cancers 
among this group as suggested by work by Shiels et al. that reported increased rates of second cancers among bladder cancer patients with a trend toward higher rates relative to current versus former smoking and magnitude of cigarettes smoked per day [21]. Prior work on second cancers in the SEER dataset [22] has not been able to define metastatic disease sites specifically as the 'lung metastasis' variable was not defined until 2010. A strength of our study is that we used more recent data after inclusion of this variable, allowing us to differentiate between metastatic bladder cancer to the lung and true primary second lung cancers. However, even though all included diagnoses were histologically confirmed, there still remains a risk of misclassification.

Additionally, we lack detailed information on the extent or intensity of staging imaging (e.g. CT or chest $\mathrm{x}$-ray) which could cause ascertainment bias. Guideline recommendations for chest imaging are only well defined for muscle invasive bladder cancer (MIBC) and the detection of metastatic disease. Screening and detection of secondary primary cancers is not well defined. Neither the European Association of Urology (EAU) nor the American Urologic Association (AUA) mentions chest imaging in the NMIBC guideline [23, 24]. Similarly, the National Comprehensive Cancer Network (NCCN) bladder cancer guideline does not recommend chest imaging for NMIBC, but does recommend imaging for MIBC with preference for chest $\mathrm{CT}$ stated at the end of the guideline [25]. Therefore, the lesser intensity of imaging use among NMIBC patients may have biased our findings towards lower detection rates. Furthermore, some data suggests that indeterminate lung nodules in patients with non-pulmonary cancer can be benign in up to $10-58 \%$ of cases, with a higher risk for a second primary cancer or metastatic lesion associated with higher pack-years, and nodule number and size [26, 27]. This further highlights the need for refined diagnostic pathways for defining metastatic lesions among bladder cancer patients regardless of disease stage.

Lastly, we did not adjust for bladder cancer treatment type, as patients who receive systemic chemotherapy or radiation may be at higher risk for second cancers than those treated with surgery or intravesical therapy alone. This lack of adjustment likely had minimal effect given the majority of the patients had NMIBC (77\%), in agreement with accepted provenances for non-invasive disease [28], a group who typically does not receive systemic chemotherapy or radiation.

\section{CONCLUSION}

In summary, we have demonstrated that all patients with bladder cancer, regardless of tumor stage, have an increased risk of synchronous and metachronous lung cancers compared to matched adults in the general population. However, among adults with MIBC, a lung nodule is more likely to be metastatic bladder cancer than a second lung primary. These findings provide data that may help inform decision making on a frequent clinical conundrum and should encourage further multi-institution assessment using data that allows adjustment for smoking status and different lung screening protocols among bladder cancer survivors.

\section{ACKNOWLEDGMENTS}

The SEER database is a population based cancer incidence and survival registry conducted by the National Cancer Institute in the United States. It covers approximately $35 \%$ of the US population. The National Cancer Institute and the SEER data managers have not verified and are not responsible for the analytic or statistical methodology used in this manuscript, or the conclusions presented by the authors. Data is publicly available and can be accessed via the SEER online request form.

\section{FUNDING}

The authors report no funding.

\section{AUTHOR CONTRIBUTIONS}

J Taylor: Performance of work, Interpretation or analysis of data, Writing the article; AB Weiner: Performance of work, Interpretation or analysis of data, writing the article; B Wang: Performance of work, Interpretation or analysis of data; A Balar: Interpretation or analysis of data; G Steinberg: Interpretation or analysis of data; R Matulewicz: Conception, Performance of work, Interpretation or analysis of data, writing the article

\section{CONFLICTS OF INTEREST}

\section{Disclosures for Gary D Steinberg:}

Is a member of Clinical Trial Protocol Committees for the following companies: Merck, BMS, Janssen, Cold Genesys, Pfizer, PhotoCure, Fidia 
He has or has been a scientific advisor/consultant within the past 5 years for the following companies: Heat Biologics, Cold Genesys, PhotoCure, Merck, Roche/Genentech, Ciclomed, Taris Biomedical, MDxHealth, Fidia Farmaceuticals, Urogen, Ferring, Aduro, Boston Scientific, Bristol Myers Squibb, Astra Zeneca, Pfizer, Janssen, Epivax Oncology, Natera, FKD, Ferring, EnGene Bio, SesenBio, BioCanCell, Nucleix, Ipsen, Combat Medical, Astellas, Fergene, Dendreon, Abbvie, Seattle Genetics.

He has equity stock/options: Epivax Oncology, Urogen

\section{Disclosures for Arjun Balar}

Genentech: Fees for Contracted Research (institution), Consultant/Advisory Role, Speaking Engagements. Incyte: Fees for Consultant/Advisory Role. Janssen: Fees for Consultant/Advisory Role. Merck: Fees for Contracted Research (institution), Consultant/Advisory Role, Speaking Engagements, Steering/Scientific Advisory Committee. Pfizer: Fees for Consultant/Advisory Role. AstraZeneca/Medimmune: Fees for Consultant/Advisory Role, Contracted Research (institution), Speaking Engagements. Nektar: Fees for Consultant Advisory Role, Contracted Research, Steering Committee Membership. Seattle Genetics: Fees for Consultant/Advisory Role, Contracted Research (institution). Immunomedics: Fees for contracted research (Institution), Fees for Consultant/Advisory Role. EpiVax Oncology: Equity, Scientific Advisory Board Member.

Jacob Taylor, Adam Weiner, Binhuan Wang and Richard S Matulewicz have nothing to disclose.

\section{SUPPLEMENTARY MATERIAL}

The supplementary material is available in the electronic version of this article: https://dx.doi.org/ 10.3233/BLC-210008.

\section{REFERENCES}

[1] Mahmud SM, Fong B, Fahmy N, Tanguay S, Aprikian AG. Effect of preoperative delay on survival in patients with bladder cancer undergoing cystectomy in Quebec: a population based study. J Urol 2006;175(1):78-83; discussion 83, 2006/1 2006.

[2] Fahmy NM, Mahmud S, Aprikian AG. Delay in the surgical treatment of bladder cancer and survival: systematic review of the literature. Eur Urol 2006;50(6):1176-82, 2006/12 2006.

[3] Curtis RE, et al. New Malignancies Among Cancer Survivors: SEER Cancer Registries 2006;1973-2000.
[4] Surveillance E. End Results (SEER) Program (www.seer. cancer.gov) SEER*Stat Database: Incidence - SEER 18 Regs Research Data+Hurricane Katrina Impacted Louisiana Cases, Nov 2018 Sub (2000-2016) Katrina/Rita Population Adjustment- Linked To County Attributes - Total U.S., 1969-2017 Counties, National Cancer Institute, DCCPS, Surveillance Research Program, released April 2019, based on the November 2018 submission.

[5] Grunfeld E, Earle CC. The interface between primary and oncology specialty care: treatment through survivorship. J Natl Cancer Inst Monogr 2010;2010(40):25-30.

[6] Kuper H, Boffetta P, Adami HO. Tobacco use and cancer causation: association by tumour type. J Intern Med 2002; 252(3):206-24.

[7] Levi F. Cancer prevention: epidemiology and perspectives. European Journal of Cancer 1999;35(14):1912-24.

[8] Strope SA, Montie JE. The causal role of cigarette smoking in bladder cancer initiation and progression, and the role of urologists in smoking cessation. J Urol 2008;180(1):31-7; discussion 37, Jul 2008.

[9] Kuo TM, Mobley LR. How generalizable are the SEER registries to the cancer populations of the USA? Cancer Causes Control 2016;27(9):1117-26.

[10] N. C. I. S. S. s. s. c. g. s. v. Surveillance Research Program.

[11] B. D. Edge SB, Compton CC, Fritz AG, Greene FL, Trotti A, N. editors. AJCC cancer staging manual (7th ed). New York, and S. 2010.

[12] Moertel CG, Dockerty MB, Baggenstoss AH. Multiple Primary Malignant Neoplasms. Cancer 1961;14:221.

[13] del Rey J, et al. Are patients with non-muscle-invasive bladder cancer a suitable population for a lung cancer screening trial? BJU Int 2010;106(1):49-52.

[14] Rusthoven KE, Flaig TW, Raben D, Kavanagh BD. High incidence of lung cancer after non-muscle-invasive transitional cell carcinoma of the bladder: implications for screening trials. Clin Lung Cancer 2008;9(2): 106-11.

[15] Sahin AF, Altok M, Akdeniz F, Yildiz G, Divrik RT. Second primary cancers in patients with urothelial cancers. Investig Clin Urol 2016;57(5):330-5.

[16] Muller J, et al. Trends in the risk of second primary cancer among bladder cancer survivors: a population-based cohort of 10047 patients. BJU Int 2016;118(1):53-9.

[17] Siegel R, Kimberly M, Jemal A. Cancer Statistics, 2020. CA: A Cancer Journal for Clinicians 2020;69(1): 7-34.

[18] Kwon WA, et al. Risk of second primary Cancer among bladder Cancer patients: a population-based cohort study in Korea. BMC Cancer 2018;18(1):617.

[19] Rasmussen LA, Jensen H, Virgilsen LF, Falborg AZ, Moller $\mathrm{H}$, Vedsted $\mathrm{P}$. Time from incident primary cancer until recurrence or second primary cancer: Risk factors and impact in general practice. Eur J Cancer Care (Engl) 2019;28(5): e13123.

[20] El-Hakim A, Smith AD, Weiss G. Patients with bladder and lung cancer: a long-term outcome analysis. BJU Int 2004;93(9):1225-7.

[21] Shiels MS, et al. Cigarette smoking prior to first cancer and risk of second smoking-associated cancers among survivors of bladder, kidney, head and neck, and stage I lung cancers. J Clin Oncol 2014;32(35):3989-95.

[22] Donin NM, Kwan L, Lenis AT, Drakaki A, Chamie K. Second primary lung cancer in United States Cancer Survivors, 1992-2008. Cancer Causes Control 2019;30(5): 465-75. 
[23] B. B. Chang SS, Chou R, et al. Treatment of non-metastatic muscle-invasive bladder cancer: AUA/ASCO/ASTRO/ SUO guideline. J Urol 2017;198:552.

[24] Babjuk M, et al. European Association of Urology Guidelines on Non-muscle-invasive Bladder Cancer (TaT1 and Carcinoma In Situ) - 2019 Update. Eur Urol 2019;76(5): 639-57.

[25] National Comprehensive Cancer Network. NCCN Clinical Practice Guidelines in Oncology: Bladder Cancer. Version 6.2020. 2020.
[26] Caparica R, et al. Pulmonary Nodules in Patients With Nonpulmonary Cancer: Not Always Metastases. J Glob Oncol 2016;2(3):138-44.

[27] Khokhar S, Vickers A, Moore MS, Mironov S, Stover DE, Feinstein MB. Significance of non-calcified pulmonary nodules in patients with extrapulmonary cancers. Thorax 2006;61(4):331-6.

[28] Taylor J, Becher E, Steinberg GD. Update on the guideline of guidelines: non-muscle-invasive bladder cancer. BJU Int 2020;125(2):197-205. 